\title{
NEW MATERIALS AND PROCESSES FOR INTEGRATED OPTICS
}

\author{
James S Wilkinson \\ Optoelectronics Research Centre \\ University of Southampton \\ Southampton \\ Hampshire \\ SO17 1BJ, UK
}

\begin{abstract}
Materials and processes for integrated optics are faced with increasing demands from telecommunications and instrumentation applications. Progress in three aspects of novel materials and processes are described; titanium-diffused sapphire waveguides and lasers, interferometric excimer laser ablation of waveguide gratings, and surface studies by waveguide surface plasmon resonance.
\end{abstract}

\section{INTRODUCTION}

Integrated optics is experiencing a period of unprecedented growth, primarily fuelled by the need for components for high-capacity optical telecommunications systems, but also stimulated by requirements for screening of chemical species for medical, environmental, and biotechnological applications. Well-established integrated optical technologies such as silica-on-silicon [1], titanium diffusion in lithium niobate [2] and ion-exchange in glass [3] provide ready solutions for many of these applications. However, the need for greater functionality in integrated optical circuits, for optical gain at new wavelengths in compact devices, and for low-cost devices, is driving research into new materials and processes for integrated optics.

In this paper, progress in research into three example systems is presented. First, the realisation of waveguides and waveguide lasers by diffusion of titanium into sapphire and the prospects for a low-threshold broadly tunable Ti:sapphire laser are discussed. Second, the writing of relief gratings in ion-exchanged glass waveguides and in thin waveguide overlayers by interferometric laser ablation is described and results are given for waveguide transmission and reflection spectra. Finally, waveguide surface plasmon resonance studies of the electrochemical underpotential deposition of a copper monolayer at the surface of a waveguide overlaid with a thin gold film are described. 


\section{TITANIUM-DIFFUSED WAVEGUIDES AND LASERS IN SAPPHIRE}

The titanium sapphire laser is a versatile laboratory tool for spectroscopic applications, with a tuning range from $650 \mathrm{~nm}$ to $1050 \mathrm{~nm}$ [4], making it well suited to tunable short-pulse operation. Realisation of a Ti:sapphire waveguide laser on a chip promises low-threshold operation for ready pumping with solidstate sources, rendering it compatible with incorporation into compact spectroscopic instruments. Further, sapphire is a versatile substrate for the growth of $\mathrm{ZnO}$ for the generation of acoustic waves, $\mathrm{GaN}$ for generation of short wavelength radiation, and silicon for realisation of electronic circuits and detectors. We have previously shown that titanium may be diffused into sapphire to give similar spectroscopic behavior to bulk-doped crystals [5], demonstrated waveguide fabrication by titanium diffusion in sapphire [6], and realised the first waveguide laser in $\mathrm{Ti}$ diffused sapphire [7]. Recently lasers with lower pump power thresholds have been produced by optimising the device geometry.

Waveguide lasers were fabricated by diffusing $270 \pm 7 \mathrm{~nm}$ thick, nominally 3 $\mu \mathrm{m}$ wide, stripes of titanium oxide, deposited by thermal evaporation from $\mathrm{Ti}_{2} \mathrm{O}_{3}$ powder, into $10 \mathrm{~mm} \times 10 \mathrm{~mm}$ c-cut sapphire wafers. Diffusion took place in an argon atmosphere at $1700 \pm 60^{\circ} \mathrm{C}$ for a duration of 60 minutes plus approximately 10 minutes each for heating and cooling. The sample was cut and polished to yield a device $4 \mathrm{~mm}$ long, and mirrors were attached to the endfaces. The mirrors had a transmission of $90 \%$ at $500 \mathrm{~nm}$ and reflectivities of $>98 \%$ at wavelengths between $760 \mathrm{~nm}$ and $810 \mathrm{~nm}$. Transverse magnetic (TM) -polarised pump radiation from an argon ion laser operating at $514.5 \mathrm{~nm}$ was chopped and end-fire coupled into a waveguide through the input mirror. The chopper operated to repeatedly pass pump radiation for $1 \mathrm{~ms}$ and block it for $19 \mathrm{~ms}$, to reduce heating of the sample. Guided output radiation was collected with a microscope objective lens and unabsorbed pump radiation was separated from laser signals using a cold mirror with an edge at $700 \mathrm{~nm}$. Using appropriate filters, the pump and signal radiation were measured using silicon photodetectors, and the waveguide mode profiles were measured using a silicon $C C D$ camera.

The waveguide laser signal output power is shown as a function of launched pump power in Figure 1 for two different channels. The launched pump power is estimated from the pump power at the output and the signal output power is averaged over 100 pump pulses for each pump power. The threshold pump power is $210 \pm 10 \mathrm{~mW}$ for both lasers and the slope efficiencies are approximately $0.06 \%$ for both lasers. Assuming that the quantum efficiency is $80 \%$ and taking the mirror transmission to be $2 \%$, the round-trip loss is estimated as $12 \mathrm{~dB}$ for both lasers. Deviations in linearity in the power characteristics are believed to be due to thermal effects. Laser spectra were measured, for laser 2 in Figure 1 using an optical spectrum analyser, with pump power $85 \mathrm{~mW}$ above threshold, and found 
to consist of two main peaks at $788 \mathrm{~nm}$ and $791 \mathrm{~nm}$. The lasers operated between $780 \mathrm{~nm}$ and $885 \mathrm{~nm}$ for different pump powers and chopper duty cycles.

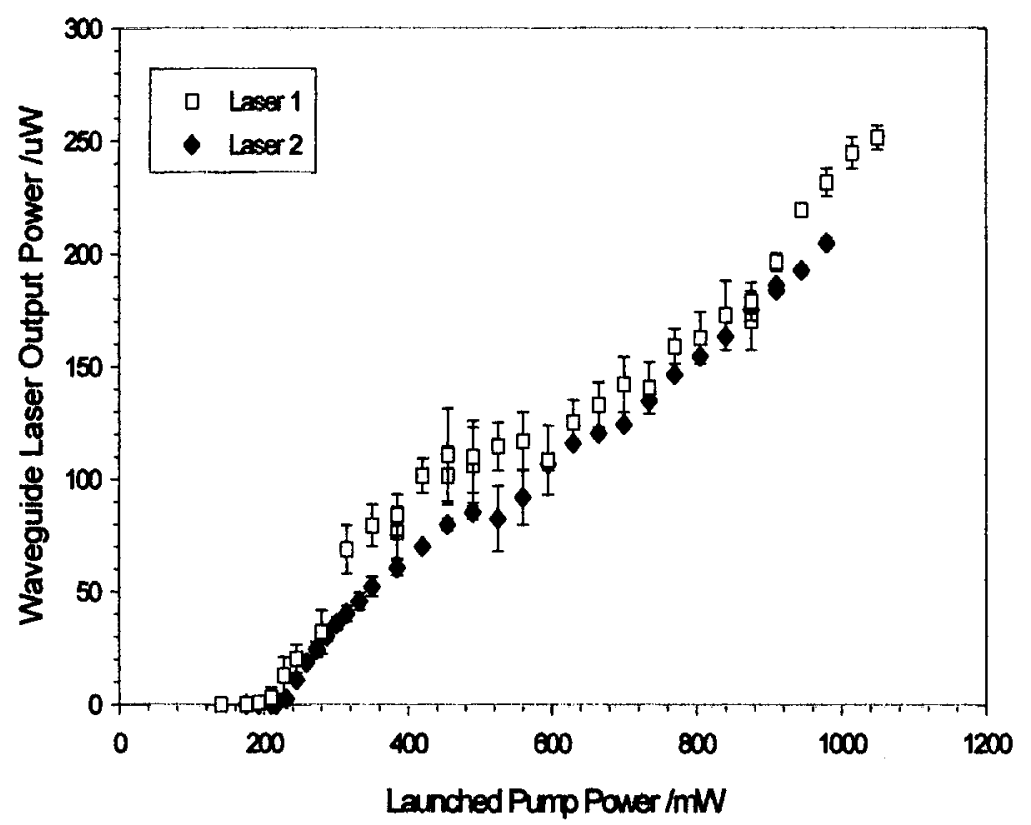

Figure 1. Ti:sapphire waveguide laser characteristics.

The mode intensity profiles for the laser mode and the pump mode for laser 1 were measured during lasing, and are shown in Figures 2 a \& b, respectively.

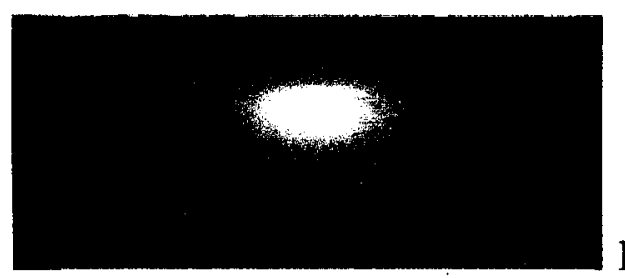

Figure 2a. Signal mode intensity profile.

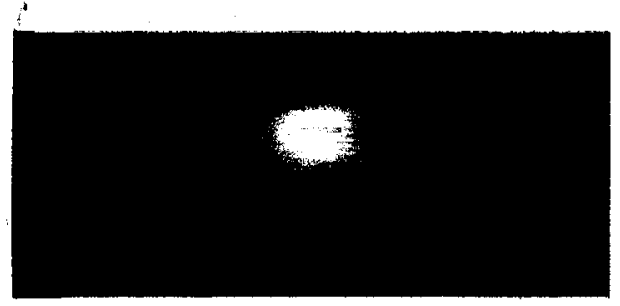

Figure $2 b$. Pump mode intensity profile. 
The intensity profiles in Figure 2 are TM polarised and show fundamental mode operation with the signal mode having approximate width of $10 \mu \mathrm{m}$ and depth of $5 \mu \mathrm{m}$ at full-width $1 / \mathrm{e}$ peak intensity. The pump mode is shown on the same scale and is substantially narrower; at wavelengths below $500 \mathrm{~nm}$ the waveguide supported more than one mode. White-light waveguide absorption measurements showed that the waveguides had an average titanium concentration equivalent to $0.13 \pm 0.02$ wt.\% $\mathrm{Ti}_{2} \mathrm{O}_{3}$ in $\mathrm{Al}_{2} \mathrm{O}_{3}$ by comparison with published results for bulk crystals [4]. These measurements yielded no evidence of significant absorption due to $\mathrm{Ti}^{4+}$, which causes absorption in the gain band and lowers the material figure of merit.

These results show a considerable reduction in pump power threshold compared with the $1.2 \mathrm{~W}$ reported previously for Ti-diffused sapphire lasers [7]. However, the low slope efficiency indicates either that the quantum efficiency is low, or that the waveguide propagation losses or mirror coupling losses are high. Future work will investigate these parameters and is expected to lead to devices with substantially lower thresholds and higher slope efficiencies. Work is also in progress to realise waveguides with smaller spotsizes, to integrate wavelength selection devices, and to operate the lasers continuously $(\mathrm{CW})$ with appropriate cooling.

\section{EXCIMER LASER ABLATION OF SURFACE WAVEGUIDE GRATINGS}

The application of excimer lasers to the fine machining of optical materials has recently been intensively investigated. Interferometric laser ablation has the potential for realising strong reflection gratings in compact waveguide devices, with the advantages of flexibility of reflection wavelength selection and minimisation of the number of process steps. We have recently demonstrated such reflection gratings near $1300 \mathrm{~nm}$, written in thallium ion-exchanged glass waveguides by interferometric laser ablation at $193 \mathrm{~nm}$ [8]. However, the exposure caused substantial damage to the waveguides, resulting in high broadband losses. Bragg gratings have been written by photolithography and etching in titanium diffused waveguides in lithium niobate overlaid with a thin silicon film to enhance modal interaction with the grating [9]. We have studied the use of thin indium tin oxide film overlayers on glass waveguides for enhancement of evanescent chemical sensing and electrochemical control of sensing reactions. Recently we have realised sub-micron relief gratings in thin $\mathrm{InO}_{\mathrm{x}}$ films overlaid on $\mathrm{K}^{+}$ion-exchanged waveguides using interferometric laser ablation at $248 \mathrm{~nm}$ [10].

Waveguides were fabricated by ion-exchange of BK7 glass in molten potassium nitrate through aluminium mask openings ranging from $3 \mu \mathrm{m}$ to $8 \mu \mathrm{m}$ at $400^{\circ} \mathrm{C}$ for 11 hours. A $135 \mathrm{~nm}$ thick film of $\mathrm{InO}_{\mathrm{x}}$ was deposited by DC magnetron sputtering in $100 \% \mathrm{O}_{2}$ to cover a length of $25 \mathrm{~mm}$ of the $40 \mathrm{~mm}$ long 
waveguides. The overlaid waveguides were exposed to a high-contrast UV fringe pattern with period $514 \mathrm{~nm}$ using the three-mirror interferometer described previously [8]. The spatial distribution of the laser output was homogenised during recording using a rotating plate. Gratings of length $16 \mathrm{~mm}$ were produced on each set of waveguides with an average pulse energy density of $60 \mathrm{~mJ} / \mathrm{cm}^{2}$ using between 5 and 100 pulses. An AFM micrograph of part of a grating ablated using 5 pulses is shown in Figure 3, where the peak to peak grating depth is 35 $\mathrm{nm}$, and scales are in nanometres.

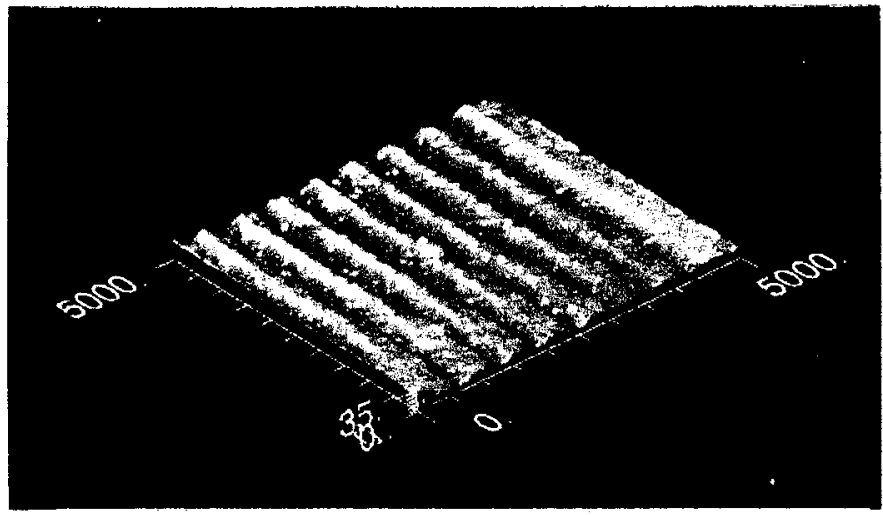

Figure 3. AFM micrograph of an ablated grating in a $135 \mathrm{~nm} \mathrm{InOx} \mathrm{film.}$

The polarised transmission spectra of the waveguides were measured by coupling the ASE spectrum of an erbium-doped fibre amplifier into each waveguide with a monomode fibre and coupling the output signal into an optical spectrum analyser using a multimode fibre. These spectra were then normalised to that obtained with the monomode fibre butted directly to the multimode fibre. Reflection spectra were obtained using a fibre coupler at the input. An example of the TE-polarised transmission and reflection spectra for an 8-micron wide waveguide overlaid with the grating shown in Figure 3 are given in Figure 4.

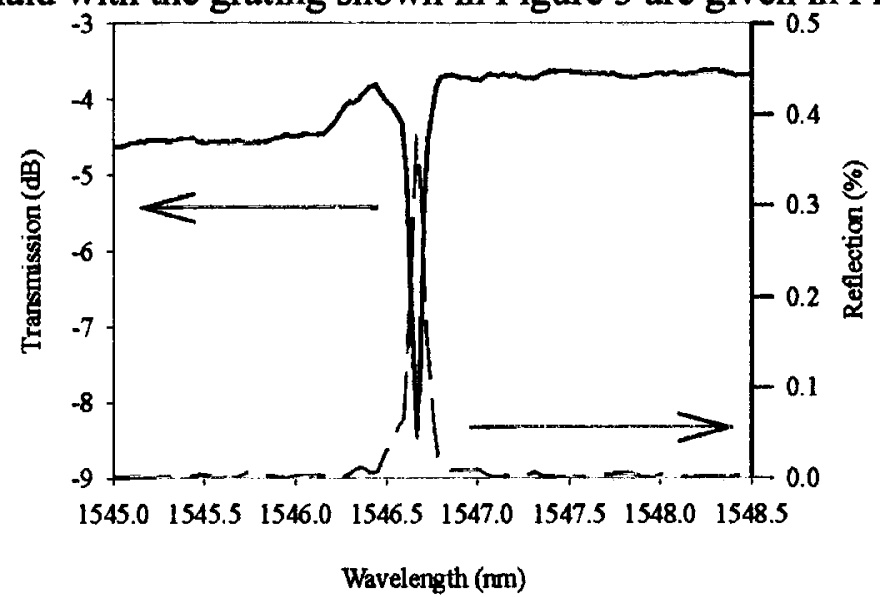

Figure 4. Waveguide transmission and reflection spectra. 
The transmission spectrum shows a broadband loss of about $4 \mathrm{~dB}$, due principally to fibre-waveguide coupling loss but also including transition losses and propagation losses in the overlayer. The grating transmission showed a clear notch at $1546.7 \mathrm{~nm}$ with a depth of approximately $4.5 \mathrm{~dB}$ and a bandwidth at fullwidth half-maximum power of $0.08 \mathrm{~nm}$. The reflection spectrum shows that reflected power is coupled into the backward-travelling waveguide mode, although the absolute value of the reflection coefficient is under further investigation. No detectable grating response was observed for the TM polarisation. The grating reflection wavelength showed a near-linear dependence upon nominal waveguide width of approximately $0.25 \mathrm{~nm} / \mu \mathrm{m}$ over the range from $3 \mu \mathrm{m}$ to $8 \mu \mathrm{m}$. Recently strong gratings have also been written by interferometric laser ablation in thin $\mathrm{Ta}_{2} \mathrm{O}_{5}$ films on glass waveguides [11].

High-quality relief gratings have been written directly in high-index overlayer films on glass waveguides. The films act both to provide a low ablation threshold material to prevent damage to the underlying waveguides and to increase the field strength at the surface, thereby increasing the overlap with the corrugation. These devices exhibit narrowband reflection of readily adjustable wavelength and strength and high polarisation selectivity, which may find application, for example, as reflectors in waveguide lasers, or in the construction of photonic crystal waveguides.

\section{SURFACE STUDIES BY WAVEGUIDE SURFACE PLASMON RESONANCE}

The potential of integrated optical surface plasmon resonance sensors has previously been demonstrated in immunosensing for waterborne pesticide analysis [12]. The combination of electrochemical techniques with waveguide sensors, through use of conducting overlayers of doped indium oxide, silver or gold, allows electrochemical control and reversal of sensing reactions and may yield complementary information on the nature of electrochemical processes at surfaces [13]. Underpotential electrochemical deposition (UPD) of monolayers of metal adsorbates on noble metal surfaces occurs below the Nernst potential required for electroplating, when the adsorbate atoms are bonded to the noble metal atoms more strongly than to a surface of the adsorbate metal [14]. Surface plasmon resonance studies of such surface reactions have great sensitivity due to the close confinement of the optical fields to the surface under investigation [15], while the waveguide configuration allows ready tailoring of the interaction strength and integration of dense arrays of such sensors through photolithographic replication.

Pyrex glass wafers $50 \mathrm{~mm}$ square were coated with an aluminium film and channels $3 \mu \mathrm{m}$ wide were opened in this film, in the form of Y-junctions as shown in Figure 5, giving rise to a diffusion mask. The coated wafer was then immersed in molten $\mathrm{KNO}_{3}$ at $389^{\circ} \mathrm{C}$ for 7.4 hours, to form potassium ion-exchanged 
waveguides, and the aluminium mask was removed. The surface of the glass was treated to promote gold adhesion by refluxing in a solution of (3-mercaptopropyl) trimethoxysilane (MPS) in water and propan-2-ol in the ratio 1:1:46 for 15 minutes.

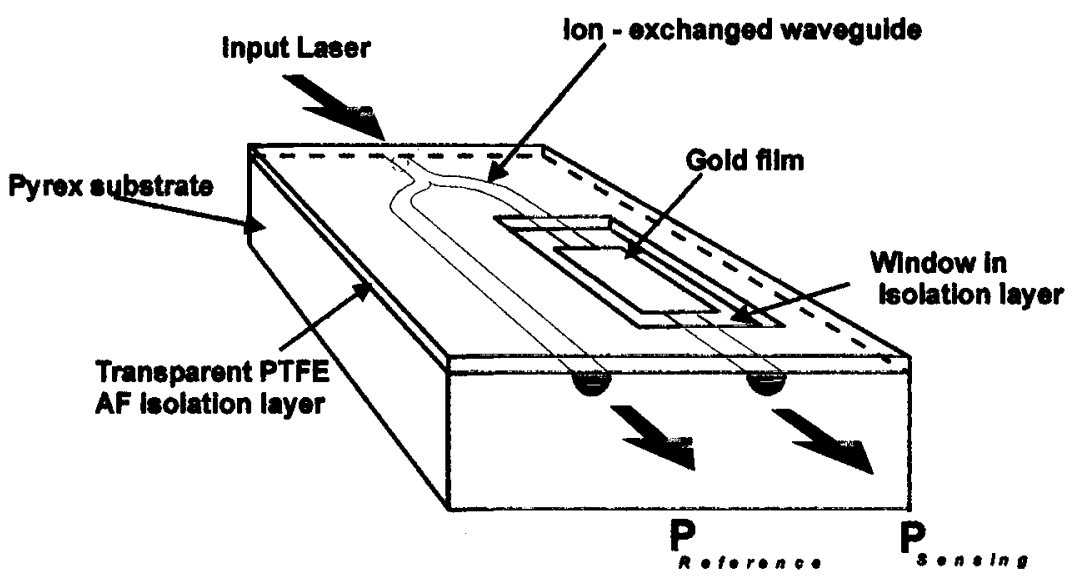

Fig (1) IOSPR Structure

Figure 5. Integrated optical surface plasmon resonance (IOSPR) sensor

The wafer was patterned with gold pads $30 \mathrm{~nm} \pm 5 \mathrm{~nm}$ thick and $3 \mathrm{~mm}$ long on one arm of each of the waveguide Y-junctions, as shown in Figure 5. The gold film serves both to guide the surface plasma wave (SPW) and as a working electrode in electrochemical studies. The second waveguide is used to provide a reference output allowing compensation for the effects of input power fluctuations, so that the device transmittivity is defined as $T=P_{\text {Reference }} / P_{\text {Sensing. }}$. The electrodes were connected by a track to pads at the edges of the substrate. Finally, a $700 \mathrm{~nm}$ thick layer of Teflon AF $1600(n \approx 1.31)$ was deposited on the surface of the substrate and patterned to form windows exposing only the gold electrodes on each Y-junction device. This device design matches the velocity of the surface plasma wave at the gold surface and the waveguide mode, for visible wavelengths at analyte indices near water, resulting in resonant coupling observed as a sharp reduction in the transmittivity [12].

A silica cell was clamped to the waveguide surface and filled with $10^{-3} \mathrm{M} \mathrm{Cu}^{2+}$ in $0.1 \mathrm{M}$ perchloric acid $(\mathrm{n}=1.334)$. A three-electrode cyclic voltammetry circuit was established by introducing a platinum wire counter electrode and a saturated calomel (SCE) reference electrode into the cell, with the gold film pads acting as the working electrodes. Permanent contact was made to the gold electrodes outside the cell at the edge of the substrate. All three electrodes were connected to a conventional potentiostat, allowing the potential of the working electrode, $\mathrm{E}$, 
with respect to the reference electrode, to be controlled and the current, I, through the working electrode to be measured.

Figure 6 shows the optical transmission and the electrochemical current against the applied potential after several potential cycles. The first part of the potential scan, from $0.5 \mathrm{~V}$ to $1.6 \mathrm{~V}$, causes the anodic oxidation of the gold surface, as shown by the current peak at about $1.1 \mathrm{~V}$ and the corresponding drop in transmission [15]. The cathodic scan shows the corresponding reduction peak in the current and increase in the optical transmission. The reduction is completed at about $0.7 \mathrm{~V}$ where the optical transmittivity has returned to its pre-oxidation value. As the potential decreases from $0.7 \mathrm{~V}$ to $0.4 \mathrm{~V}$, the transmittivity increases due to the alteration of the ionic distribution of the double layer at the metal electrolyte interface. This ionic redistribution involves the chemisorption of anions at the gold, and results in a small displacement current. At a potential of $0.24 \mathrm{~V}$ a peak in the current is observed, which is due to the deposition of a monolayer of $\mathrm{Cu}$ onto the gold film surface. The deposition of this monolayer of approximate thickness $0.3 \mathrm{~nm}$ gives rise to a $10 \%$ drop in the optical transmittivity. Integration of the peak current over time during deposition gives a charge of $0.41 \mathrm{mC} / \mathrm{cm}^{2}$, which is close the theoretical value of $0.44 \mathrm{mC} / \mathrm{cm}^{2}$ for a monolayer of $\mathrm{Cu}^{2+}$ ions transferring two electrons per ion to an ideal $\mathrm{Au}$ (111) surface. The direction of the potential scan is reversed at $0.1 \mathrm{~V}$ and a peak in current is observed at $0.29 \mathrm{~V}$, corresponding to stripping of the $\mathrm{Cu}$ monolayer, and the transmittivity increases correspondingly.

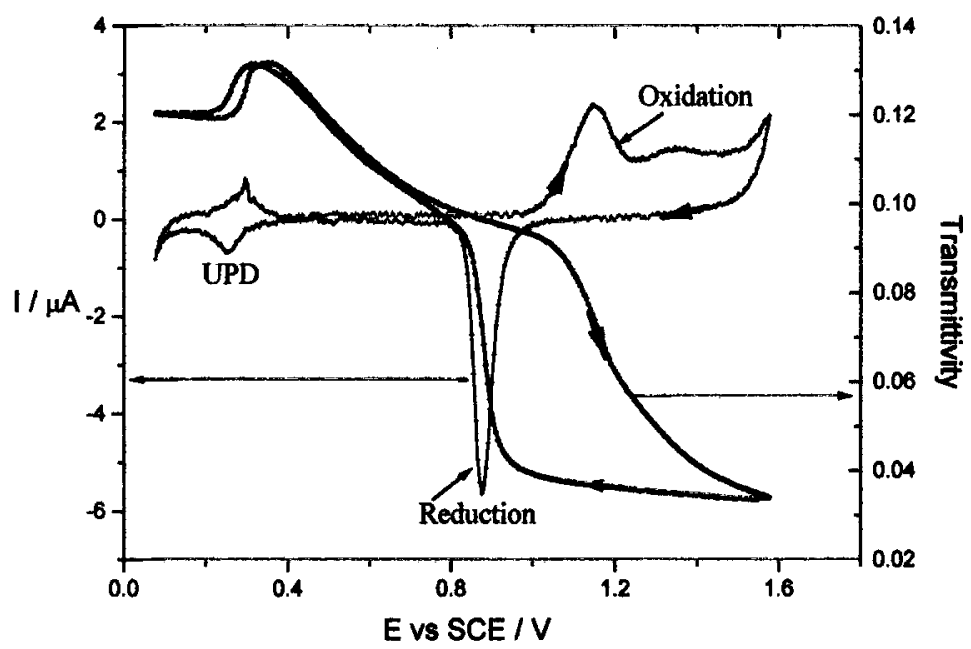

Fig (3) Optical and Electrochemical response to UPD of copper and the oxidation of a gold film.

Figure 6. Optical and electrochemical response of IOSPR sensor. 
The processes involved in the electrochemical oxidation and reduction of a gold surface and the underpotential deposition of $\mathrm{Cu}^{2+}$ on gold may be continuously monitored in-situ with high sensitivity using a compact integrated optical surface plasmon resonance sensor. The waveguide approach is ideally suited for multisensor integration and the results compare favourably with those obtained using ellipsometry and reflectance measurements [16,17]. Sensor arrays for interrogation of surface films are expected to find wide application in chemical sensing applications, and combined monitoring of electrochemical and optical parameters provides a powerful combination for multiparameter surface sensing.

\section{CONCLUSION}

Growth in telecommunications applications of integrated optics has stimulated the exploitation of planar optical waveguides in a wide range of fields. Many new applications require materials with different properties to conventional waveguide materials, new fabrication processes, or new techniques to characterise surface processes. An example of progress in research in each of these three areas has been described. Work on sapphire waveguides has been primarily concerned with realisation of lasers, which show potential as compact, low-cost, low-power devices; however, other applications in high-temperature or hostile environments can be foreseen. Interferometric excimer laser ablation of thin film waveguide overlays has been shown to be a flexible technique for production of strong highquality reflection gratings for wavelength selection in integrated devices. Finally, interfacial studies using integrated optical surface plasmon resonance device have been described, where the surface sensitivity of planar waveguides is exploited to monitor electrochemical processes with great sensitivity .

\section{ACKNOWLEDGEMENTS}

The author would like to thank the many colleagues who have contributed to the work of the Integrated Optics \& Microstructures Group at Southampton University. The Optoelectronics Research Centre is an interdisciplinary centre supported by the UK Engineering and Physical Sciences Research Council.

\section{REFERENCES}

1. A.Kaneko, T.Goh, H.Yamada, T.Tanaka \& I.Ogawa, "Design and applications of silica-based planar lightwave circuits", IEEE Journal of Selected Topics in Quantum Electronics, 5 [5] 1227-1236 (1999).

2. R.Wessel, R.Ricken, K.Rochhausen, H.Suche \& W.Sohler, "Supermode stabilised coupled-cavity 5- and $10-\mathrm{GHz}$ mode-locked $\mathrm{Ti}: \mathrm{Er}: \mathrm{LiNbO}_{3}$ waveguide lasers", IEEE J. Quantum Electronics, QE-36 [3] 394-399 (2000). 
3. N.V.Nikonorov \& G.T.Petrovskii, "Ion-exchanged glasses in integrated optics: The current state of research and prospects (a review)", Glass Physics \& Chemistry, 25 [1] 16-55 (1999).

4. P F Moulton, "Spectroscopic Properties and Laser Characteristics of Ti: $\mathrm{Al}_{2} \mathrm{O}_{3}$ ", J. Opt. Soc. Am. B, 3 125-133 (1986).

5. L.M.B.Hickey, E.Martins, J.E.Román, W.S.Brocklesby \& J.S.Wilkinson, "Fluorescence of $\mathrm{Ti}^{3+}$ ions thermally diffused into sapphire", Optics Letters, 21 [8] 597-599 (1996).

6. L.M.B.Hickey \& J.S.Wilkinson, "Titanium diffused waveguides in sapphire", Electronics Letters, 32 2238-2239 (1996).

7. L.M.B.Hickey, A.A.Anderson \& J.S.Wilkinson, "Ti-sapphire channel waveguide laser by thermal diffusion of titanium in sapphire", Proc. 8th European Conference on Integrated Optics, Stockholm, Paper PD6 (1997).

8. S.Pissadakis, L.Reekie, M.Hempstead, M.N.Zervas \& J.S.Wilkinson, "Relief gratings on $\mathrm{Er} / \mathrm{Yb}$-doped borosilicate glasses and waveguides by excimer laser ablation", Applied Surface Science, 153 200-210 (2000).

9. C.P.Hussel \& R.V.Ramaswamy, "High-index overlay for high-reflectance DBR gratings in $\mathrm{LiNbO}_{3}$ channel waveguides", Phot. Tech. Lett. 9 636-638 (1997)

10. S.Pissadakis, L.Reekie, M.N.Zervas, J.S.Wilkinson, K.Moschovis \& G.Kiriakidis, "High-index overlay gratings on $\mathrm{K}^{+}$- exchanged waveguides in BK-7 glass using excimer laser ablation", CLEO 2000, San Francisco, (2000).

11. S.Pissadakis, L.Reekie, J.S.Wilkinson \& G.Kiriakidis, "Sub-micron period grating structures in $\mathrm{Ta}_{2} \mathrm{O}_{5}$ and $\mathrm{InO}_{\mathrm{x}}$ thin oxide films, fabricated using $248 \mathrm{~nm}$ interferometric excimer laser ablation", CLEO Europe 2000, Nice, (2000).

12. R.D. Harris, B.J. Luff, J.S. Wilkinson, A. Brecht, G. Gauglitz, R.A. Abuknesha, "Integrated optical surface plasmon resonance immunoprobe for simazine detection", Biosensors and Bioelectronics, 14 377-387 (1999).

13. C.R.Lavers, R.D.Harris, S.Hao, J.S.Wilkinson, K.O'Dwyer, M.Brust \& D.J.Schiffrin, "Electrochemically-controlled waveguide-coupled surface plasmon sensing", Journal of Electroanalytical Chemistry, 387 11-22 (1995).

14. J.G. Xu and X.W. Wang, "Study of copper underpotential deposition on Au(111) surfaces", Surface Science, 408 317-325 (1998).

15. Y.Iwasaki, T. Horiuchi, M.Morita \& O. Niwa, "Time differential surface plasmon resonance measurements applied for electrochemical analysis", Electroanalysis, 9 1239-1241 (1997).

16. W. Visscher and A.P Cox, "Ellipsometry of metal deposition", Electrochimica Acta, 37 2245-224 (1992).

17. K. Takamura, F. Watanabe and T. Takamura, "An interpretation on the origin of the specular reflectance change caused by the submonolayer formation of foreign metals on some precious metal electrodes", Electrochimica Acta, 26 $979-987$ (1981). 